\title{
Função executiva em idosos: um estudo utilizando subtestes da Escala WAIS-III
}

\author{
Eliane Ferreira Carvalho Banhato ${ }^{1}$ \\ Elizabeth do Nascimento
}

\begin{abstract}
Resumo
Avaliar funções executivas é relevante por apresentarem declínio precoce com a idade. Disponibilizar instrumentos para pesquisa e avaliação de idosos é necessário em face do aumento dessa população. Este estudo transversal objetivou avaliar funções executivas de idosos utilizando cinco subtestes da WAIS-III, identificando associações com idade, gênero e escolaridade. A amostra comunitária constituiu-se de 346 idosos com média etária de 74,14 anos (DP $=8,49)$ e escolaridade de 7,2 anos (DP $=4,5)$. Mediu-se validade de construto com testes de Fluência Verbal (convergente) e CES-D (divergente). Univariadas determinaram diferença significativa segundo gênero, com melhor desempenho masculino. Idade correlacionou-se negativa e significativamente com tarefas executivas. Escolaridade afetou a velocidade de processamento, mas não a atenção, raciocínio e organização perceptovisuomotora. Concluiu-se pela adequação dos subtestes para avaliação executiva de idosos. Recomenda-se estabelecimento de pontos de corte para possibilitar diferenciação entre envelhecimento normal e patológico.

Palavras-chave: Funções executivas; Escala WAIS-III; Envelhecimento.
\end{abstract}

\begin{abstract}
Evaluating executive functions is relevant because of their presenting premature decline with age. It's necessary to make instruments for research and evaluation of elderly people available due to the increase of this population. This transversal study had as its objective the evaluation of executive functions of elderly people using five sub tests of WAIS-III identifying associations with age, gender and level of instruction. The communitarian sample consisted of 346 elderly people with average age of 74,14 years $(\mathrm{DP}=8,49)$ and level of instruction of 7,2 years $(\mathrm{DP}=4,5)$. The validity of construct was measured with tests of Verbal Fluency (convergent) and CES-D (divergent). Univariate Analysis have determined significant difference according to gender, with better masculine performance. Age correlated negatively and significantly to executive tasks. Level of instructions affected the processing speed, but not the attention, reasoning and visual, motor and perceptive organization. The study conclusion was in favor of the adequacy of the sub tests for executive evaluation of elderly people. The establishment of limit points is recommended in order to make the differentiation between normal and pathological aging possible.
\end{abstract}

Executive functions in elderly: A study using WAIS-III Subtests Scale

Keywords: Executive functions; WAIS-III Scale; Aging.

\section{Introdução}

O termo funções executivas (FE) designa os processos cognitivos de controle e integração destinados à execução de um comportamento dirigido a objetivos, necessitando de subcomponentes como atenção, programação e planejamento de seqüências, inibição de processos e informações concorrentes e monitoramento. O lobo frontal, particularmente a região pré-frontal, tem sido relacionado com essas funções (Kristensen, 2006).

No envelhecimento, tanto normal quanto patológico, as funções executivas tendem a estar prejudicadas. No envelhecimento normal, as alterações executivas ocorrem de modo gradual e lento até os 60 anos, tornando-se mais aceleradas a partir dos 70 anos.
Uma possível explicação para esse declínio é o desgaste fisiológico natural dos lobos frontais (Woodruff-Pak, 1997). Também no comprometimento cognitivo leve (CCL) e nas demências, as alterações de natureza executiva estão presentes, porém de forma precoce e quantitativamente mais intensa.

A demência é uma doença neurodegenerativa progressiva e, até o momento, inexorável. Seu diagnóstico, segundo o Manual diagnóstico e estatístico de transtornos mentais (DSM-IV) (2003), é feito na presença de déficit de memória e de outra função cognitiva, associada a um comprometimento do funcionamento social e ocupacional. O tipo mais prevalente é a demência de Alzheimer, cuja taxa de incidência praticamente dobra a cada cinco anos de idade (Lopes, Bottino \& Hototian, 2006).

\footnotetext{
${ }^{1}$ Endereço para correspondência:

Av. Vasconcelos, 50 apto. 1.400 - Alto dos Passos - 36026-480 - Juiz de Fora-MG

E-mail: ebanhato@powerline.com.br
} 
Por outro lado, o comprometimento cognitivo leve (CCL) é um estado intermediário entre o envelhecimento normal e a demência e se caracteriza pela queixa e déficit de memória, porém sem dificuldades nas atividades de vida diária e com funcionamento intelectual geral normal. Apesar de não estar ligado à doença, pessoas com CCL apresentam alto risco de desenvolver demência (Bottino \& Moreno, 2006).

Segundo Jacobson, Delis, Bondi e Salmon (2002), o declínio executivo pode preceder o início de uma demência em sete a dez anos. Essa característica pode destacar as funções executivas como marcadoras no diagnóstico diferencial entre demências e envelhecimento normal, tornando relevante sua investigação.

Dentre as técnicas empregadas para rastrear o funcionamento cerebral, ressalta-se a avaliação neuropsicológica, que fundamentada no princípio de interdependência entre cérebro e comportamento, examina o indivíduo de forma global (história pessoal, familiar, social e médica) e específica (funções cognitivas e emocionais), por meio de testes psicometricamente qualificados e padronizados para o contexto em que serão utilizados. O estudo de revisão de Petersen e colaboradores (2001) recomenda a utilização tanto de instrumentos de rastreio quanto de baterias flexíveis para a identificação e o monitoramento de pessoas com algum tipo de comprometimento cognitivo. Para tanto, a adaptação de instrumentos psicométricos a determinado contexto se faz necessária, principalmente, em razão da heterogeneidade cultural e educacional.

No entanto, muitos testes utilizados em avaliações psicológicas ou neuropsicológicas são antigos, importados ou não-validados para a população brasileira (Alchieri, 2004). Especificamente a testagem em idosos apresenta um aspecto complicador. Dentre os testes que apresentam validade no Brasil, a maioria não está normatizada para o grupo etário acima de 60 anos ou, quando está, a coorte investigada é muito pequena.

Entre os testes de rastreio cognitivo global, o Miniexame do Estado Mental - MEEM (Folstein, Folstein \& Mc Hugh, 1975) é o mais amplamente utilizado (Nitrini e cols., 2005). Já as funções executivas são investigadas em nosso meio principalmente pelo Teste do Desenho do Relógio e de Fluência Verbal (FV). Apesar de ter aplicação simples, o teste FV é uma tarefa cognitiva complexa e muito sensível a todo tipo de dano cerebral, apontando, precocemente, os processos de deterioração executiva. Dentre as diferentes possibilidades, o teste de FV mais utilizado é o de categoria semântica (animais/minuto), tendo sido recomendado pelo Departamento Científico de Neurologia Cognitiva e do Envelhecimento, da Academia Brasileira de Neurologia (Nitrini e cols., 2005).
Um instrumento flexível considerado padrão-ouro de avaliação intelectual é a Escala Wechsler de Inteligência para Adultos (WAIS) (Viana \& Koenig, 2002). Em sua versão mais recente - WAIS-III (Wechsler, 1997), foram introduzidas mudanças estruturais importantes (ampliação dos limites de idade e inclusão de três novos subtestes) que permitem a avaliação de componentes cognitivos específicos, como funções executivas, linguagem, memória. Além disso, é particularmente importante o fato dessa escala ter sido normatizada para o contexto brasileiro (Nascimento, 2000). Os parâmetros psicométricos adequados obtidos em sua validação demonstraram que a WAIS-III pode ser recomendada na avaliação de habilidades cognitivas de adultos brasileiros.

Um aspecto importante em relação à idade deve ser considerado quando da utilização da escala WAIS-III. Apesar de as normas elaboradas para a escala terem contemplado adultos com idades até 89 anos, estes foram distribuídos em apenas dois grupos de idosos: 60 a 64 anos; e 65 até 89 anos. Se considerarmos que um idoso de 65 anos tende a ser muito diferente de outro com 75 ou 85 anos, conclui-se que as faixas etárias são muito amplas, não permitindo acompanhamento sistemático do perfil cognitivo no curso da vida. Dessa forma, estudos que investiguem aspectos específicos da cognição focalizando faixas etárias mais estreitas podem contribuir tanto para o melhor aproveitamento da escala WAIS-III em idosos quanto para aquisição de mais conhecimento sobre o processo cognitivo na velhice.

Além da forma de aplicação da escala total (14 subtestes), a literatura apresenta formas alternativas, onde se elegem subtestes que contemplem o domínio a ser investigado (Donders \& Axelrod, 2002; López, González Vilariño \& Linares, 2003; Wymer, Rayls \& Wagner, 2003). Segundo os autores, essa estratégia resulta em redução de 20 a 50\% do tempo de aplicação (López e cols., 2003). Esse recurso é particularmente útil no caso de amostra idosa. As médias do tempo de aplicação de 60 a 90 minutos obtidas em amostra de adultos jovens tendem a ser excessivamente mais elevadas em pessoas mais velhas. Tal fato pode provocar fadiga, dispersão atencional e baixa motivação para colaborar, comprometendo a eficácia da avaliação.

Outro importante fator a ser considerado na avaliação psicológica é a possível interferência de algumas variáveis no critério diagnóstico. Uma delas é a escolaridade, que parece ser influenciada tanto pela idade com que o indivíduo começou a estudar quanto pela freqüência à escola e número de anos estudados. Para Meguro e colaboradores (2001), o nível educacional elevado seria fator preventivo no embotamento do estado mental durante o envelhecimento normal. 
Outras variáveis intervenientes seriam a idade e o gênero. Estudos apontam para uma relação inversamente proporcional entre idade e desempenho intelectual. No entanto, essa influência não está bem determinada em decorrência das limitações metodológicas utilizadas (Salthouse, 1994).

No que se refere ao gênero, as evidências empíricas são contraditórias. Colom, Quiroga e JuanEspinosa (1999) afirmaram que as mulheres apresentam melhor desempenho nas habilidades verbais, enquanto os homens vão melhor em tarefas visuoconstrutivas e matemáticas.

Porém, avaliando a inteligência fluida em jovens estudantes de ambos os sexos, Colom e García-López (2001) não encontraram diferença estatisticamente significativa. O estudo de Brucki e Rocha (2004), que investigaram a influência do gênero, idade e escolaridade no desempenho executivo de idosos, encontrou melhor desempenho dos homens em teste de Fluência Verbal, apesar da diferença ter sido significativa $(p<0,05)$ apenas em um dos intervalos de tempo avaliados (30-45 segundos).

Pelo exposto, e diante do acelerado crescimento da população idosa no contexto brasileiro, verifica-se a grande importância de disponibilizar instrumentos adequados tanto para a pesquisa quanto para a avaliação neuropsicológica de idosos, principalmente em funções marcadoras de processos patológicos, como as habilidades executivas. Este estudo pretende contribuir nesse mister, uma vez que teve como objetivo avaliar o perfil em habilidades executivas de diferentes faixas etárias de idosos da comunidade, utilizando subtestes específicos da escala WAIS-III. Buscou-se, ainda, identificar possíveis associações entre o desempenho executivo e idade, gênero e escolaridade.

\section{Método}

\section{Participantes}

Para seleção da amostra utilizou-se o critério de aleatorização ( $40 \%$ da amostra) de um banco de dados do Estudo dos Processos de Envelhecimento Saudável (PENSA). A amostra foi estratificada com base em variáveis demográficas, incluindo idade, sexo e nível educacional. Com relação à variável idade, a amostra total foi dividida em 6 grupos etários: 60-64, 65-69, 70-74, 7579, 80-84 e maiores de 85 anos. A variável escolaridade foi estratificada em três categorias: analfabetos, os que possuíam de 1 a 7 anos de estudos e aqueles com 8 ou mais anos de escolaridade. Os critérios de inclusão utilizados foram: fazer parte da $1^{\text {a }}$ etapa do estudo PENSA; apresentar boa capacidade de discriminação visual e auditiva e assinar o Termo de Consentimento Livre e Esclarecido.

\section{Procedimentos}

Este estudo foi aprovado pelo Comitê de Ética em Pesquisa (COEP) sob número 120/2005. Os indivíduos selecionados foram contatados por telefone e convidados a participar de uma avaliação neuropsicológica. Quando aceitavam, era agendada uma visita à residência dos participantes, que assinavam o Termo de Consentimento Livre e Esclarecido. A aplicação dos testes teve duração aproximada de 40 minutos. A coleta de dados ocorreu entre dezembro de 2004 e abril de 2005.

\section{Instrumentos}

Escala Wechsler de Inteligência para Adultos - WAISIII - $3^{\text {a }}$ edição: Adaptada, validada e normatizada para contexto brasileiro por Nascimento (2000). Dos 14 subtestes que constituem a escala, foram selecionados, por meio de consulta a especialistas e à literatura, os que medem funções executivas e atenção. Três fazem parte da escala de execução (Raciocínio Matricial, Procurar Símbolos e Cubos) e um é componente da escala verbal (Dígitos). A medida de desempenho para todos os subtestes foi o escore bruto obtido na avaliação. Os subtestes utilizados são descritos a seguir.

Raciocínio Matricial: formado por 26 itens, a tarefa consiste em completar um padrão a partir de cinco alternativas apresentadas. Investiga o processamento da informação visual e o raciocínio abstrato e é relativamente livre da cultura e da linguagem. O exame é suspenso após 4 erros consecutivos na versão adaptada.

Procurar Símbolos: a tarefa consiste em identificar, num limite de 120 segundos, se os símbolos mostrados no grupo-alvo estão presentes no grupo de busca. Constituído por 60 itens, esse subteste investiga a atenção e rapidez do processamento mental.

Cubos: esse subteste consiste em reproduzir 14 modelos apresentados como padrões com cubos geométricos, bidimensionais e bicolores, e apresenta tempo-limite de execução em todos os níveis. Investiga as habilidades de organização perceptual e visual, a conceitualização abstrata, a visualização espacial, a inteligência fluida e a velocidade de processamento mental, e tem aplicação suspensa após 3 erros consecutivos.

Dígitos: consiste na repetição oral de seqüências numéricas em ordem direta (16 itens) e inversa (14 itens), perfazendo 30 pontos. Esse subteste investiga as habilidades de recordação e repetição imediata (memória de trabalho). A suspensão da tarefa é feita após 2 erros dentro de uma mesma série de repetição. 
Teste de Fluência Verbal (categoria animais): utilizado como instrumento auxiliar de avaliação executiva. Avalia funções executivas, memória semântica e linguagem. Consiste em propor ao examinando que, num período de 1 minuto, diga o mais rápido que puder nomes de animais que conseguir se lembrar. $\mathrm{O}$ ponto de corte sugerido é 9 para escolaridade baixa (menor que 8 anos) e 13 para os de 8 ou mais anos de estudo (Nitrini e cols., 2005).

CES-D - Center Epidemiologic Survey Depression - (Radloff, 1977): escala de rastreamento populacional para detectar sintomas depressivos em populações adultas. É composta de 20 itens relativos a humor, comportamento e percepção, considerados relevantes em estudos clínicos sobre depressão. As respostas são dadas de acordo com a freqüência (nunca, raramente, às vezes, freqüentemente, sempre) com que cada sintoma esteve presente na semana anterior à aplicação do instrumento.

Análise estatística: Realizou-se análise bivariada de Pearson para avaliar a validade convergente dos subtestes estudados em relação a outras formas de medida já existentes para o mesmo construto. Para investigar a validade convergente utilizou-se o Teste de Fluência Verbal. Como os subtestes utilizados por este estudo foram construídos para avaliar habilidade atentiva, visuoconstrutiva e de memória de curto prazo, hipotetizou-se que esses obtivessem correlação com o Teste de Fluência Verbal, que investiga os mesmos construtos. A validade discriminante foi testada em relação à escala de depressão CES-D. Validada para populações adultas da comunidade, a CES-D demonstrou ser um instrumento confiável para investigação de transtornos depressivos e, portanto, a hipótese do estudo é que não seriam encontradas correlações significativas com os subtestes da WAIS-III.

O Teste $t$ de Student foi realizado para comparar os resultados executivos com a variável gênero. Análises univariadas (ANOVA) foram utilizadas para avaliar os efeitos da idade e da escolaridade nos escores brutos dos subtestes da WAIS-III. Comparações post hoc de Bonferroni (1935) foram realizadas. Os dados foram analisados com o auxílio do software SPSS, versão 10.0.

\section{Resultados}

\section{Características da amostra}

Participaram deste estudo 346 idosos, sendo $74,1 \%(n=256)$ do gênero feminino. A idade variou entre 61 a 100 anos, com média de 74,14 anos (DP = 8,5) e mediana igual a 73 anos. A distribuição etária mostrou que 36,1\% $(n=125)$ eram idosos jovens (60-69 anos), 38,2\% $(n=132)$ eram idosos médios (70-79 anos) e $25,7 \%(n=89)$ eram idosos-idosos (maior que 80 anos). Em relação ao estado civil, 167 idosos (48,4\%) eram casados, 136 viúvos (39,4\%), 23 solteiros (6,7\%), e 19 separados (5,5\%). A média de anos estudados foi 7,2 anos $(4,5)$ e mediana igual a 6 . Cerca de $10 \%$ da amostra declarou possuir nível superior completo. Quando solicitados a avaliar a saúde, 244 idosos (69,5\%) responderam muito boa, $94(26,8 \%)$ como regular e 13 idosos $(3,7 \%)$ avaliaram-na como ruim. Todos os idosos pertenciam à zona urbana. A Tabela 1 apresenta a distribuição da amostra segundo grupo etário e nível educacional.

Tabela 1 - Caracterização da amostra

\begin{tabular}{|c|c|c|}
\hline Variáveis & $\mathrm{F}$ & $\%$ \\
\hline \multicolumn{3}{|c|}{ Faixa etária em anos } \\
\hline $60-64$ & 56 & 16,2 \\
\hline $65-69$ & 76 & 22,0 \\
\hline $70-74$ & 65 & 18,8 \\
\hline $75-79$ & 62 & 17,9 \\
\hline$\geq 80$ & 87 & 25,1 \\
\hline \multicolumn{3}{|l|}{ Gênero } \\
\hline Masculino & 90 & 26,0 \\
\hline Feminino & 256 & 74,0 \\
\hline \multicolumn{3}{|c|}{ Anos de estudo } \\
\hline Analfabetos & 9 & 2,6 \\
\hline 1 a 7 anos & 194 & 56,1 \\
\hline Maior igual a 8 anos & 143 & 41,3 \\
\hline
\end{tabular}




\section{Validade de construto}

Para verificar a validade dos subtestes como instrumento de avaliação executiva, realizou-se a validade de construto convergente e discriminante. A validade convergente foi realizada entre os subtestes da escala WAIS-III utilizados neste estudo e o Teste Fluência Verbal (Tabela 2). Todos os subtestes da escala WAIS-III se correlacionaram entre si $(\phi \leq 0,01)$, demonstrando que os subtestes captaram aspectos de um mesmo construto (função executiva). O Teste
Fluência Verbal se correlacionou positiva e significativamente com Raciocínio Matricial $(r=0,38$; $p$ $\leq 0,01)$, Procurar Símbolos $(r=0,48 ; p \leq 0,01)$, Cubos $(r=0,31 ; p \leq 0,01)$ e Dígitos total $(r=0,32 ; p \leq 0,01)$.

A validade discriminante foi realizada com a escala de depressão CES-D. Não houve correlação estatisticamente significativa entre os subtestes e a referida escala, exceto pelo subteste Procurar Símbolos que, embora marginalmente, associou-se negativa e significativamente $(r=-0,170 ; p<0,05)$.

Tabela 2 - Matriz de correlações entre subtestes WAIS-III, FV e CES-D

\begin{tabular}{lccccc}
\hline & $R M$ & $P S$ & $C U B$ & $D I G$ & $F V$ \\
\hline PS & $0,57^{* *}$ & - & - & - & - \\
CUB & $0,50^{* *}$ & $0,46^{* *}$ & - & - & - \\
DIG & $0,34^{* *}$ & $0,34^{* *}$ & $0,62^{* *}$ & - & - \\
FV & $0,38^{* *}$ & $0,45^{* *}$ & $0,31^{* *}$ & $0,32^{* *}$ & - \\
CES-D & $-0,10$ & $-0,17^{*}$ & $-0,12$ & $-0,08$ & $-0,04$ \\
\hline
\end{tabular}

$* p<0,05 ; * * p<0,01$

Legenda: RM (Raciocínio Matricial); PS (Procurar Símbolos); CUB (Cubos); DIG (Dígitos); FV (Fluência Verbal).

\section{Habilidades cognitivas}

O desempenho executivo avaliado pelos subtestes da escala WAIS-III, relativo ao raciocínio abstrato (Raciocínio Matricial), à organização visuoespacial (Cubos), às habilidades atentivas e de memória de curto prazo (Dígitos) e à velocidade de processamento da informação (Procurar Símbolos) foram investigados tanto de forma geral quanto segundo as variáveis gênero, idade e escolaridade. Os resultados estão relacionados na Tabela 3.

Em razão da escala WAIS-III não apresentar ponto de corte específico, utilizou-se o procedimento de comparar as médias ponderadas obtidas pelos idosos nos subtestes com as do grupo de referência (20-34 anos) apresentadas no manual (Wechsler, 2004). Esse grupo foi selecionado como referência fundamentada no argumento de ser este o período da vida onde se obteriam as melhores performances cognitivas.

As médias nas tarefas que avaliam raciocínio abstrato $(\mathrm{M}=7,50 ; \mathrm{DP}=4,15)$, organização visuoespacial $(\mathrm{M}=17,08 ; \mathrm{DP}=8,18)$ e velocidade de processamento $(\mathrm{M}=10,71 ; \mathrm{DP}=6,66)$ mostraram-se notadamente mais baixas que as do grupo de referência. Por outro lado, as tarefas propostas para avaliação da memória de trabalho e atenção $(\mathrm{M}=11,47 ; \mathrm{DP}=3,55)$ demonstraram médias semelhantes às do grupo de referência (Tabela 3).

Tabela 3 - Desempenho cognitivo da amostra nos subtestes da WAIS-III

\begin{tabular}{|c|c|c|c|c|c|c|c|c|c|}
\hline \multirow{2}{*}{\multicolumn{2}{|c|}{ Escore de referência* }} & \multicolumn{2}{|c|}{$\begin{array}{c}\mathrm{RM} \\
12-14\end{array}$} & \multicolumn{2}{|c|}{$\begin{array}{c}\text { PS } \\
23-25\end{array}$} & \multicolumn{2}{|c|}{$\begin{array}{c}\text { Cubos } \\
27-30\end{array}$} & \multicolumn{2}{|c|}{$\begin{array}{c}\text { Dígitos } \\
12-13\end{array}$} \\
\hline & & Média & $\mathrm{DP}$ & Média & $\mathrm{DP}$ & Média & DP & Média & $\mathrm{DP}$ \\
\hline \multirow[t]{2}{*}{ Gênero } & Masculino & 8,55 & 4,86 & 12,21 & 6,79 & 19,10 & 9,00 & 12,96 & 3,82 \\
\hline & Feminino & 7,15 & 3,83 & 10,17 & 6,54 & 16,26 & 8,11 & 10,92 & 3,29 \\
\hline \multirow{6}{*}{ Faixa etária } & $60-64$ & 8,24 & 4,62 & 13,19 & 6,53 & 15,67 & 7,03 & 12,16 & 3,62 \\
\hline & $65-69$ & 9,00 & 4,46 & 13,70 & 6,39 & 17,39 & 7,70 & 12,12 & 3,09 \\
\hline & $70-74$ & 8,31 & 4,48 & 12,25 & 6,60 & 16,98 & 8,72 & 12,08 & 3,70 \\
\hline & $75-79$ & 6,93 & 3,25 & 8,97 & 6,63 & 16,85 & 7,76 & 11,63 & 3,61 \\
\hline & $80-84$ & 5,36 & 3,19 & 6,29 & 3,44 & 18,16 & 10,27 & 9,97 & 3,83 \\
\hline & $\geq 85$ & 5,35 & 2,66 & 6,36 & 4,31 & 17,39 & 8,20 & 9,76 & 2,83 \\
\hline \multirow[t]{4}{*}{ Escolaridade } & Analfabetos & 5,43 & 3,91 & 7,20 & 4,44 & 21,00 & 8,89 & 10,86 & 4,63 \\
\hline & 1- 7anos & 7,20 & 3,95 & 10,37 & 6,40 & 16,36 & 7,72 & 11,32 & 3,26 \\
\hline & $\geq 8$ anos & 8,03 & 4,38 & 11,29 & 7,03 & 17,89 & 8,69 & 11,70 & 3,87 \\
\hline & Amostra geral & 7,50 & 4,15 & 10,71 & 6,66 & 17,08 & 8,18 & 11,47 & 3,55 \\
\hline
\end{tabular}

*Escore de referência $=$ escore bruto obtido pelo grupo de referência (20-34 anos) correspondente ao resultado ponderado igual a 10 . 


\section{Efeito do gênero}

O gênero afetou o desempenho executivo dos idosos, tendo os homens obtido médias significativamente superiores às mulheres nas tarefas que avaliaram atenção $\mathrm{e}$ memória de curto prazo $(t=4,50, p=0,00)$, velocidade de processamento da informação $(t=2,44, p=0,01)$, memória verbal $(t=3,717, p=0,00)$, raciocínio abstrato $(t$ $=9,66, p<0,01)$ e organização visuoespacial $(t=2,47, p$ $<0,01)$.

\section{Efeito da idade}

A idade se correlacionou negativa $\mathrm{e}$ significativamente com funções executivas. A análise ANOVA e a comparação post hoc demonstraram diferenças estatisticamente significativas entre os idosos mais jovens (60-69) e os mais idosos (80 ou mais anos) nas habilidades de raciocínio abstrato $(F=7,61, p=0,00)$, organização perceptovisuomotora $(F=5,78, p=0,00)$, velocidade do processamento da informação $(F=14,90, p=0,00)$ e atenção e memória de trabalho $(F=4,32, p=0,00)$.

\section{Efeito da escolaridade}

Em relação à escolaridade, os idosos com maior índice educacional apresentaram melhores desempenhos, apesar de as médias serem mais baixas que as do grupo de referência. Para testar a hipótese de que mais anos de escolaridade serviriam de fator protetor no declínio cognitivo, realizou-se análise ANOVA. As médias nas tarefas de velocidade de processamento da informação, obtidas pelo subteste Procurar Símbolos $(F=4,166, p=$ $0,02)$ sofreram influência do nível educacional. A comparação post hoc revelou diferença significativa entre o grupo de analfabetos e os de 1 a 7 anos de estudo $(p=$ $0,05)$ e entre os primeiros com os que estudaram 8 ou mais anos. As tarefas de raciocínio abstrato apontaram uma tendência de efeito da escolaridade $(F=2,41, p=0,09)$. Não foi observado efeito da escolaridade em tarefas que mediram memória de trabalho e atenção $(F=0,23, p=$ $0,79)$ e organização perceptovisuomotora $(F=0,427, p=$ $0,65)$.

\section{Discussão}

A heterogeneidade e o aumento crescente da população idosa tornam relevante a avaliação de funções cognitivas desse segmento populacional a fim de assegurar melhor qualidade para essa fase da vida. A avaliação neuropsicológica pode detectar processos patológicos bem como os déficits precoces. Este estudo privilegiou as funções executivas por ser uma das primeiras habilidades a declinarem no envelhecimento e por se tratar de uma amostra comunitária e, portanto, presumivelmente preservados cognitivamente.
A utilização de subtestes de uma escala de padrão internacional de referência (WAIS-III) demonstrou a adequação dos mesmos às diferentes faixas etárias que compõem a etapa da vida denominada velhice. Comparações estatisticamente significativas entre teste específico de medida executiva (Fluência Verbal) e os subtestes da escala WAIS-III sugeriram que os últimos estiveram mensurando de fato o que se propuseram a medir. No entanto, as forças de associação moderadas entre eles permitiram inferir que talvez os construtos avaliados sejam semelhantes, mas não os mesmos. Esse achado aponta para um evento psicométrico comum, ou seja, a dificuldade de se encontrarem testes específicos para avaliar construtos iguais. Uma outra possibilidade para tal resultado pode ser decorrente da técnica de associação empregada. Como a correlação de Pearson mede apenas associação linear, os efeitos não-lineares não foram representados no coeficiente de correlação.

Além das dificuldades metodológicas, outro importante ponto a ser considerado refere-se à questão conceitual. A literatura apresenta divergências quanto a unidade ou diversidade das funções executivas. Duncan (1995, citado por Stuss \& Alexander, 2000), por exemplo, defende o caráter unitário das funções executivas enquanto função única da inteligência fluida (gf) com supervisão exercida pelos lobos frontais. Por outro lado, há autores como Stuss e Alexander (2000), que argumentam que o termo funções executivas engloba uma multiplicidade de funções refinadas e complexas que estariam relacionadas a diferentes regiões dentro dos lobos frontais, e destas com outras regiões cerebrais. Assim, tarefas simples, como de um teste cognitivo, não captariam toda a complexidade desse sistema supramodal de processamento múltiplo (Salthouse, Atkinson \& Berish, 2003; Stuss \& Alexander, 2000).

A validade discriminante demonstrou que os subtestes da WAIS-III não correlacionaram com as medidas de sintomas depressivos, com exceção do subteste que avalia a velocidade do processamento de informações. Ainda que a literatura aponte para o declínio da velocidade de reação associada à idade, em deprimidos essa habilidade pode estar mais comprometida. No entanto, cabe ressaltar que a força de correlação entre as variáveis foi muito fraca, o que pode significar uma associação não-genuína.

Comparados com as médias do grupo de referência (20-34 anos), os escores ponderados dos idosos nos subtestes executivos foram inferiores, apontando para uma perda leve na memória de trabalho e moderada nas habilidades atencionais, na velocidade de processamento das informações e na organização visuoespacial. A constatação de que a amostra apresentava independência nas atividades básicas e instrumentais de vida diárias

Psico-USF, v. 12, n. 1, p. 65-73, jan./jun. 2007 
permitiu confirmar a precocidade do declínio de funções executivas consoante o relatado na literatura (Jacobson e cols., 2002). Esse achado demonstra a necessidade de acompanhamento contínuo das características psicológicas dos indivíduos com piores performances, de modo a permitir identificação diferencial dos casos que seguirão o envelhecimento normal daqueles que evoluirão para processos demenciais, patologia de alta prevalência nos dias atuais.

Diversas variáveis apresentaram influência no desempenho executivo de idosos. Vale ressaltar a influência do gênero nas performances executivas, com os homens apresentando melhores desempenhos. Além desses resultados poderem estar associados a uma particularidade da coorte histórica, também apontaram para a evidência de que os homens, em média, neste contexto cultural específico, apresentariam maior desenvolvimento das habilidades abstratas e visuoespaciais. Resultados similares foram relatados por Colom, Quiroga e Juan-Espinosa (1999) e Colom e García-López (2001), que argumentaram que a diferença entre gênero poderia estar associada aos estereótipos dos papéis sexuais e das normas diferenciadas por sexo do contexto social.

A idade exerceu influência significativa nos desempenhos executivos, atenção e memória de trabalho, sendo a velocidade de processamento da informação a mais prejudicada, ou seja, os idosos mais velhos necessitaram mais tempo para alcançar o mesmo nível de acurácia no desempenho da tarefa. Esse resultado é similar aos encontrados tanto em estudos longitudinais (Schaie, 1996) quanto transversais (Kaufman, 2001; Wecker, Kramer, Wisniewsky, Delis \& Kaplan, 2000).

Quanto à escolaridade, o presente estudo observou diferença significativa na velocidade do processamento das tarefas entre os idosos, dependendo do nível de escolaridade. De modo geral, a educação formal facilita o desempenho intelectual, ou seja, há correlação positiva entre essas variáveis. Segundo Mortiner (1988), a educação provocaria um efeito protetor contra demências, principalmente na demência de Alzheimer. Por outro lado, a escolaridade não apresentou efeitos na performance executiva de repetição, organização perceptovisuomotora e atenção. Uma possível razão para isso seria a nãolinearidade na relação entre escolaridade e funções de execução (Ardila, Ostrosky-Solis, Rosseli \& Gómez, 2000). Segundo os autores, a escolaridade apresentaria substancial correlação em testes de conteúdo verbal, mas não estaria sistematicamente relacionada aos de resolução de problemas, ou seja, aos critérios funcionais da inteligência (funções executivas). Um outro fator que pode ter influenciado a ausência de efeito dos anos estudados seria o elevado percentual de indivíduos (37\%) com alto nível Psico-USF, v. 12, n. 1, p. 65-73, jan./jun. 2007 educacional da amostra em estudo (acima de 8 anos), o que poderia representar um viés de amostragem.

Quanto à utilização dos subtestes da WAIS-III na investigação de funções executivas, os resultados do presente estudo permitiram demonstrar que foram úteis em apontar declínios. Dentre as vantagens observadas, cita-se a reconhecida validade e confiabilidade da escala, o menor tempo de aplicação do instrumento e a facilidade de comparação com outros estudos por se tratar de um instrumento amplamente difundido em nível internacional.

No entanto, é importante ressaltar que a nãodiscriminação em faixas etárias mais detalhadas a partir dos 65 anos, bem como a inexistência de pontos de corte dos subtestes da escala WAIS-III, limitam-na como instrumento de detecção de declínios patológicos. Estudos futuros com amostras clínicas tornam-se imprescindíveis para aumentar a precisão na diferenciação diagnóstica, tendo em vista estabelecer pontos de corte para processos demenciais.

\section{Referências}

Alchieri, J. C. (2004). Aspectos instrumentais e metodológicos da avaliação psicológica. Em V. M. Andrade, F. H. Santos, O. F. A. Bueno, Neuropsicologia hoje (pp. 13-36). Porto Alegre: Artes Médicas.

Ardila, A., Ostrosky-Solis, F., Rosselli M. \& Gómez, C. (2000). Age-related cognitive decline during normal aging: The complex effect of education. Archives of Clinical Neuropsychology, 15, 495-514.

Bonferroni, C. E. (1935). Il calcolo delle assicurazioni su gruppi di teste, Studi in Onore del Professore Salvatore Ortu Carboni. Rome, 13-60.

Bottino, C. M. C. \& Moreno, M. D. P. (2006). Comprometimento cognitivo leve: critérios diagnósticos e validade clínica. Em C. M. C. Bottino, J. Laks \& S. L. Blay (Orgs.). Demência e transtornos cognitivos em idoso (pp. 31-37). Rio de Janeiro: Guanabara Koogan.

Brucki, S. M. \& Rocha, M. S. G. (2004). Category fluency test: effects of age, gender and education on total scores, clustering and switching in Brazilian Portuguese-speaking subjects. Brazilian Journal of Medical and Biological Research, 37(12), 1771-1777.

Colom, R. \& García-López, O. (2001). Sex differences in fluid intelligence among high school graduates. Personality and Individual Differences, 32, 445-451.

Colom, R., Quiroga, M. H. \& Juan-Espinosa, M. (1999). Are cognitive sex differences disappearing? Evidence from Spanish populations. Personality and Individual Differences, 27, 1189-1195. 
Donders, J. \& Axelrod, B. N. (2002). Two-subtest estimations of WAIS-III factor index scores. Psychological Assessment, 14, 360-364.

DSM-IV-TRTM (2003). Manual diagnóstico e estatístico de transtornos mentais. $4^{\mathrm{a}}$ ed. Porto Alegre: Artes Médicas.

Folstein, M. F., Folstein, S. E. \& Mc Hugh, P. R. (1975). Mini-mental state: A practical method for grading the cognitive state of patients for the clinician. Journal of Psychiatry, 12, 189-198.

Jacobson, M. W., Delis, D. C., Bondi, M. W. \& Salmon, D. P. (2002). Do Neuropsychological Tests detect preclinical Alzheimer's disease: individual-test versus cognitive-discrepancy score analyses. The American Psychological Associations, 16(2), 132-139.

Kaufman, A. S. (2001). WAIS-III: IQs, Horn's theory, and generational changes from young adulthood to old age. Science Direct, 29(2), 131-167.

Kristensen, C. H. (2006). Funções executivas e envelhecimento. Em M. A. M. P. Parente (Orgs.). Cognição e envelhecimento (pp. 97-111). Porto Alegre: Artmed.

Lopes, M. A., Bottino, C. M. C. \& Hototian, S. R. (2006). Epidemiologia das demências: análise crítica das evidências atuais. Em C. M. C. Bottino, J. Laks \& S. L. Blay (Orgs.). Demência e transtornos cognitivos em idoso (pp. 23-30). Rio de Janeiro: Guanabara Koogan.

López. M. J. L., González, J. M. R., Vilariño, C. S. \& Linares, E. T. (2003). Utilidad de las formas cortas de la Escala de Inteligencia de Wechsler para Adultos (WAIS). Anales de Psicología, Murcia (España): Servicio de Publicaciones de la Universidad de Murcia, 19(1), 53-63.

Meguro, K., Shimada, M., Yamagucho S., Ishii, H., Shimada, Y., Sato, M., Yamadori A. \& Sekita,Y. (2001). Cognitive function and frontal lobe atrophy in normal elderly adults: Implications for dementia not as aging-related disorder and the reserve hypothesis. Psychiatric Clinic Neuroscience, 55(6), 565-72.

Mortiner, J. A. (1988). Do psychosocial risk factors contribute to Alzheimer's type? Chichester: John Wiley and Sons.

Nascimento, E. (2000). Adaptação e validação do teste WAIS-III para um contexto brasileiro (Tese de Doutorado). Brasília: Universidade de Brasília.

Nitrini, R., Caramelli, P., Bottino, C. M. C., Damasceno, B. P., Brucki, S. M. D. \& Anghinah, R. (2005). Diagnóstico de doença de Alzheimer no Brasil: avaliação cognitiva e funcional. Recomendações do
Departamento Científico de Neurologia Cognitiva e do Envelhecimento da Academia Brasileira de Neurologia. Arquivos de Neuro-Psiquiatria, 63(3A), 720727.

Petersen, R. C., Steves, J. C., Ganguli, M., Tangalos, E. G., Cummings, J. L. \& DeKosky, S. T. (2001). Practice parameter: early detection of dementia, mild cognitive impairment (an evidence-bases review). Neurology, 56, 1133-1142.

Radloff, L. S. (1977). The CES-D scale: a self-report depression scale for research in the general population. Applied Psychological Measurement, 1, 385401.

Salthouse, T. A., Atkinson, T. M. \& Berish, D. E. (2003). Executive functioning as a potential of agerelated cognitive decline in normal adults. Journal of Experimental Psychology, 132(4), 566-594.

Salthouse, T. A. (1994). The aging of working memory. American Psychological Association, 8(4), 535-543.

Schaie, K. W. (1996). Intellectual development in adulthood: The Seatle longitudinal study. New York: Cambridge University Press.

Stuss, D. T. \& Alexander, M. P. (2000). Executive functions and the frontal lobes: a conceptual view. Psychological Research, 63, 289-298.

Viana, E. B. \& Koenig, A. M. (2002). Avaliação cognitiva. Em E. V. Freitas, L. Py, A. L. Neri, F. A. X. Cançado, M. L. Gorzoni \& S. M. Rocha (Orgs.). Tratado de geriatria e gerontologia (pp. 921-928). Rio de Janeiro: Guanabara Koogan.

Wechsler, D. (1997). Wechsler Adult Intelligence Scale-III (Third edition). Nova York: Psychological Corporation.

Wecker, N., Kramer, J., Wisniewsky, A., Delis, D. \& Kaplan, E. (2000). Age effects on executive ability. Neuropsychology, 14(3), 409-414.

Woodruff-Pak, D. S. (1997). The Neuropsychology of aging. Malden: Blackwell Publishers.

Wymer, J. H., Rayls, K. \& Wagner, M. T. (2003). Utility of a clinically derived abbreviated form of the WAISIII. Archives of Clinical Neuropsychology, 18(8), 917-927.

Recebido em junho de 2006 Reformulado em março de 2007 Aprovado em abril de 2007 
Sobre as autoras:

Eliane Ferreira Carvalho Banhato é mestre em Psicologia do Desenvolvimento pela Universidade Federal de Minas Gerais, MG, especialista em Gerontologia pela Sociedade Brasileira de Geriatria e Gerontologia, professora da Especialização em Desenvolvimento Humano do Departamento de Psicologia da Universidade Federal de Juiz de Fora e professora do curso de Graduação em Psicologia do Centro de Estudo Superior CES, JF.

Elizabeth do Nascimento é doutora em Psicologia pela Universidade de Brasília, DF, professora do Curso de Graduação e do Programa de Pós-Graduação do Departamento de Psicologia da UFMG e membro fundador do Laboratório de Avaliação das Diferenças Individuais (LADI). 
